\title{
Mise en évidence et évaluation de la quantité de lait de vache dans les fromages de brebis par isoélectrofocalisation des lactosérums. Application au cas de fromages très protéolysés : fromages type Roquefort
}

\author{
S Rispoli 1, M Rivemale 2, R Saugues 1 \\ 1 Laboratoire interrégional de la direction générale de la concurrence, de la consommation \\ et de la répression des fraudes, 2, rue Saint-Pierre, BP 2042, 34024 Montpellier Cedex; \\ 2 Centre de recherche, société des Caves, 12250 Roquefort, France
}

(Reçu le 20 août 1990; accepté le 14 mai 1991)

\begin{abstract}
Résumé - Le protocole précédemment décrit pour rechercher et doser le lait de vache dans les fromages au lait de brebis (Rispoli et Saugues, 1989) est appliqué ici au cas des fromages très protéolysés, tels que les fromages à pâte persillée. Pour cette étude, une fabrication spéciale de fromages de brebis fraudés par ajout de lait de vache (cru, pasteurisé, poudre) est réalisée par la Société des caves Roquefort, en utilisant la technologie type Roquefort. Ces fromages utype Roquefort" sont analysés par isoélectrofocalisation des lactosérums, à divers stades d'affinage (de 15 à 365 jours après la fabrication). Malgré le degré de protéolyse avancé de ces fromages, dans tous les cas le lait de vache est mis en évidence et la quantité est déterminée.
\end{abstract}

isoélectrofocalisation / lactosérum / fromage au lait de mélange / fromage Roquefort

Summary - Identification and quantitative determination of cow's milk in ewe's cheeses using isoelectric focusing of wheys. Application to cheeses at high ripening stage: Roquefort cheeses. The procedure previously described by Rispoli and Saugues (1989) for qualitative and quantitative determination of cow's milk in ewe's cheeses was applied to highly ripened cheeses such as blue cheese. Special fabrication of reference ewe's cheeses adulterated with cow's milk (raw, pasteurized or dried) was made by the Société des Caves Roquefort according to Roqueforttype technology. These Roquefort-type cheeses were analyzed by isoelectric focusing of extracted cheese wheys at different ripening stages (15-365 d after fabrication). In spite of the high ripening stage of these cheeses, the qualitative and quantitative determination of cow's milk was still possible. 


\section{INTRODUCTION}

L'adultération du lait de brebis par ajout de lait de vache lors de la fabrication des fromages de brebis constitue une fraude. Pour mettre en évidence cette fraude, parmi les différentes méthodes existant actuellement, les techniques électrophorétiques (électrophorèse, isoélectrofocalisation) sont très utilisées (Ramos et Juarez, 1984). Elles permettent de caractériser les protéines des différentes espèces de lait.

Ces méthodes donnent des résultats satisfaisants pour l'analyse des laits et des fromages peu affinés, mais présentent des limites d'utilisation pour les fromages à forte maturation. En effet, dans ce cas, des produits issus de la protéolyse peuvent masquer les bandes protéiques à identifier, rendant ainsi le diagramme électrophorétique ininterprétable (Pierre et Portmann, 1970; Krause et al, 1982).

Parmi les fromages de brebis, les fromages Roquefort se distinguent par leur niveau élevé de protéolyse : les enzymes de la moisissure Penicillium exercent une action protéolytique profonde, libérant des produits de poids moléculaires, aussi bien élevés que faibles (Desmazeaud et al, 1976); ce type de fromage constitue donc un matériel idéal pour tester les limites de validité de telles méthodes en ce qui concerne la protéolyse. Si la méthode de Addeo et al (1984), basée sur la caractérisation des paracaséines $\kappa$ par isoélectrofocalisation permet d'analyser les fromages Pecorino jusqu'à 5 mois d'affinage, cette même méthode n'a pas permis d'analyser les fromages type Roquefort à forte protéolyse.

En effet, Mauriello et al (1989) dosent le lait de vache dans des fromages type Roquefort jusqu'à 1 mois, par isoélectrofocalisation des paracaséines $\kappa$, mais pour un temps d'affinage plus long (fromages de 4 et 5 mois), cette méthode s'est avérée inadaptée pour ce type de fromage : une bande résultant de la protéolyse se confond alors avec la paracaséine $\kappa$ bovine.

Les méthodes basées sur la caractérisation des protéines du lactosérum ont été moins étudiées que celles s'intéressant aux caséines. Cet intérêt moindre peut s'expliquer par leur limite d'utilisation en cas de traitement thermique, du fait de la thermosensibilité de ces protéines (Donovan et Mulvihill, 1987).

Dans le présent travail, nous nous proposons de rechercher et quantifier le lait de vache dans des fromages type Roquefort par isoélectrofocalisation des lactosérums. Le protocole précédemment décrit par Rispoli et Saugues (1989) sera utilisé, afin d'en apprécier la limite de validité en cas de protéolyse.

\section{MATÉRIEL ET MÉTHODES}

\section{Laits mis en fabrication}

- lait de brebis cru, race Lacaune (lait de grand mélange).

- lait de vache cru (lait de grand mélange).

- lait de vache pasteurisé $\left(70^{\circ} \mathrm{C}\right.$ pendant $\left.25 \mathrm{~s}\right)$.

- lait de vache en poudre (fabrication "spray") reconstitué à $12 \%(P / N)$.

\section{Fabrication des fromages}

Plusieurs types de laits entrent dans la fabrication des fromages :

- lait $100 \%$ de brebis;

- lait de brebis contenant $4 \%$ de lait de vache cru;

- lait de brebis contenant $4 \%$ de lait de vache pasteurisé; 
- lait de brebis contenant $7 \%$ de lait de vache pasteurisé;

- lait de brebis contenant $5 \%$ de lait de vache en poudre reconstitué.

A partir de ces laits, la Société des Caves de Roquefort réalise des minifabrications de fromages, selon la technologie suivante : au lait réchauffé à $33^{\circ} \mathrm{C}$ sont ajoutés les ferments lactiques, la présure et le Penicillium; après $2 \mathrm{~h}$, le caillé est découpé, brassé et mis en moule; l'égouttage s'effectue en salle à $18^{\circ} \mathrm{C}$ pendant 3 jours; les fromages sont ensuite salés au sel sec; après le salage, soit 10 jours après la fabrication, ils sont transférés en cave d'affinage.

L'affinage comporte deux phases : la $1^{\text {re }}$ phase dure 15 jours à $12{ }^{\circ} \mathrm{C}$, le Penicillium se développe dans les cavités de la pâte; la seconde phase s'effectue à basse température pendant une durée variable (de 3 mois à $1 \mathrm{an}$ ).

Dans l'étude présente, un fromage de chaque minifabrication est prélevé pour l'analyse à divers temps d'affinage : selon les cas, 15, 70, 140,180 et 365 jours après la fabrication (tableaux I et II).

\section{Détermination de la teneur en matières azotées des fromages}

L'azote total exprimé en protéines est déterminé par la méthode de Kjeldahl $(\mathrm{N} \times 6,38)$. L'azote soluble est déterminé de la même manière sur le filtrat obtenu, après précipitation à l'acide trichloroacétique du fromage solubilisé en milieu citrate de sodium.

\section{Préparation des extraits pour I'isoélectrofocalisation (IEF)}

Cinq grammes de fromage (prélevés dans la partie contenant le moins de moisissures) sont broyés dans 5 à $10 \mathrm{ml}$ d'eau distillée. Le mélange est ajusté à $\mathrm{pH} 4,6$, puis centrifugé pendant 15 min à $6800 \mathrm{~g}$. Le surnageant contenant les protéines du lactosérum est récupéré : les traces de matière grasse sont éliminées par un traitement au chloroforme, à raison de $1 \mathrm{ml}$ de lactosérum pour $2 \mathrm{ml}$ de solvant.

\section{Protocole d'analyse par isoélectrofocalisation (IEF)}

Les gels pour l'IEF sont réalisés selon la méthode précédemment décrite par Rispoli et Saugues (1989): gel de 0,8 mm d'épaisseur à $5 \%$ d'acrylamide, $0,15 \%$ bisacrylamide, $2 \%$ d'ampholyte $\mathrm{pH}$ 4-8.

Sur un même gel, sont déposés les extraits des fromages Roquefort à analyser et des fromages témoins $(100 \%$ brebis, 5,10 et $100 \%$ vache). La migration a lieu à $8^{\circ} \mathrm{C}$, à une puissance constante de $5 \mathrm{~W}$ pendant $2 \mathrm{~h} 30$. Le gel est coloré selon la méthode de Blakesley et Boezi (1977).

A l'aide des témoins, nous repérons sur le diagramme IEF les bandes $V$ et $B$ choisies pour caractériser les deux espèces de lait. L'intensité de coloration de ces bandes est évaluée à l'aide d'un densitomètre TLC Scanner II Camag; la lecture s'effectuant au centre des bandes colorées. Chaque profil d'IEF est analysé 3 fois par densitométrie; pour chaque lecture densitométrique, le rapport de densité de bandes v/b est calculé.

Tous les fromages à analyser sont traités 3 fois, conformément au protocole ci-dessus décrit (fig 1).

mube

Pour chaque fromage analysé, nous obtenons 9 valeurs du rapport v/b. La moyenne de ce rapport est reportée sur l'abaque (fig 2) précédemment établi (Rispoli et Saugues, 1989) et le pourcentage de lait de vache est déterminé.

\section{RÉSULTATS}

\section{Analyse de la matière azotée des fromages}

Les teneurs en matière azotée totale et matière azotée soluble de tous les fromages Roquefort analysés sont rassemblées dans le tableau I. Nous voyons que le taux de matière azotée soluble par rapport à la matière azotée totale, qui n'est que de $8 \%$ pour un fromage frais, augmente au cours de l'affinage. Après 70 
Tableau I. Teneur en azote total et en azote soluble des fromages Roquefort.

Total nitrogen and soluble nitrogen in Roquefort cheeses.

\begin{tabular}{|c|c|c|c|c|}
\hline $\begin{array}{l}\text { Échantillons } \\
\text { analysés }\end{array}$ & $\begin{array}{l}\text { Temps } \\
\text { d'affinage } \\
\text { (jours) }\end{array}$ & $\begin{array}{l}\text { Azote total exp } \\
\text { en protéines } \\
\quad(g / 100 \mathrm{~g})\end{array}$ & $\begin{array}{c}\text { Azote soluble exp } \\
\text { en protéines } \\
(g / 100 \mathrm{~g})\end{array}$ & $\begin{array}{c}\% \text { Azote soluble } \\
\text { azote total }\end{array}$ \\
\hline $100 \%$ brebis & 15 & 19,4 & 1,7 & 8,7 \\
\hline $4 \%$ vache cru & 15 & 19,3 & 1,8 & 9,3 \\
\hline $5 \%$ vache poudre & 15 & 19,5 & 1,9 & 9,7 \\
\hline $7 \%$ vache pasteurisé & 15 & 18,4 & 1,8 & 9,8 \\
\hline $100 \%$ brebis & 70 & 19,6 & 6,8 & 34,7 \\
\hline $4 \%$ vache pasteurisé & 70 & 19,1 & 9,0 & 47,1 \\
\hline $5 \%$ vache poudre & 70 & 20,6 & 9,9 & 48,0 \\
\hline $7 \%$ vache pasteurisé & 70 & 18,9 & 9,6 & 50,8 \\
\hline $100 \%$ brebis & 140 & 20,2 & 11,3 & 55,9 \\
\hline $4 \%$ vache cru & 140 & 19,9 & 12,6 & 63,3 \\
\hline $5 \%$ vache poudre & 140 & 20,3 & 12,8 & 63,0 \\
\hline $7 \%$ vache pasteurisé & 140 & 19,5 & 11,4 & 58,4 \\
\hline $100 \%$ brebis & 180 & 19,9 & 10,2 & 51,3 \\
\hline $4 \%$ vache pasteurisé & 180 & 18,3 & 10,7 & 58,5 \\
\hline $7 \%$ vache pasteurisé & 180 & 19,1 & 10,5 & 55,0 \\
\hline $100 \%$ brebis & 365 & 20,0 & 12,5 & 62,5 \\
\hline $4 \%$ vache pasteurisé & 365 & 20,0 & 13,3 & 66,5 \\
\hline
\end{tabular}

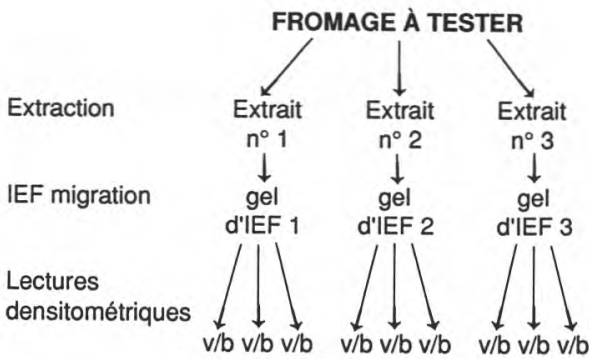

Fig 1. Pour chaque fromage analysé, nous obtenons 9 yaleurs du rapport v/b. La moyenne de ce rapport est reportée sur l'abaque (fig 4) précédemment établie (Rispoli et Saugues,

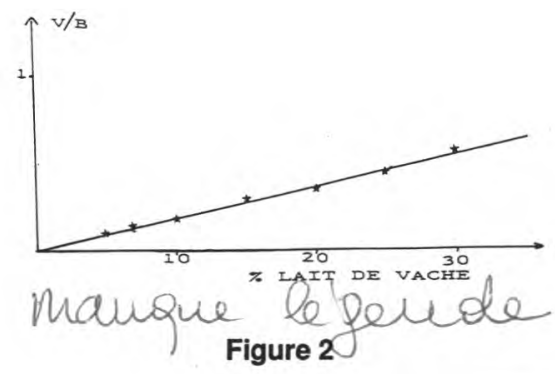
1989) et le pourcentage de lait de vache est déterminé. 
Tableau II. Pourcentages de lait de vache déterminés en fonction des rapports de densité de bandes $\mathrm{v} / \mathrm{b}$ pour tous les fromages analysés.

Percentages of cow's milk determined by density coloration ratios of the cle bands for all analysed cheeses.

\begin{tabular}{|c|c|c|c|c|}
\hline \multirow[t]{2}{*}{$\begin{array}{l}\text { Échantillons } \\
\text { analysés }\end{array}$} & \multirow[t]{2}{*}{$\begin{array}{l}\text { Temps d'affinage } \\
\text { (jours) }\end{array}$} & \multicolumn{2}{|c|}{$\begin{array}{l}\text { Rapport de densité de } \\
\text { bande } v / b\end{array}$} & \multirow[t]{2}{*}{$\begin{array}{c}\% \text { de lait } \\
\text { de vache }\end{array}$} \\
\hline & & Moyenne (a) & Écart type & \\
\hline Pur brebis & 15 & \multicolumn{2}{|c|}{ Lait de vache non décelé } & \\
\hline $4 \%$ vache cru & 15 & 0,050 & 0,020 & 3 \\
\hline $5 \%$ vache poudre & 15 & 0,056 & 0,015 & 3 \\
\hline $7 \%$ vache pasteurisé & 15 & 0,130 & 0,031 & 7 \\
\hline Pur brebis & 70 & \multicolumn{2}{|c|}{ Lait de vache non décelé } & \\
\hline $4 \%$ vache pasteurisé & 70 & 0,063 & 0,023 & 3 \\
\hline $5 \%$ vache poudre & 70 & 0,063 & 0,018 & 3 \\
\hline $7 \%$ vache pasteurisé & 70 & 0,130 & 0,032 & 7 \\
\hline Pur brebis & 140 & \multicolumn{2}{|c|}{ Lait de vache non décelé } & \\
\hline $4 \%$ vache cru & 140 & 0,080 & 0,022 & 4 \\
\hline $5 \%$ vache poudre & 140 & 0,090 & 0,018 & 5 \\
\hline $7 \%$ vache pasteurisé & 180 & 0,146 & 0,020 & 7 \\
\hline Pur brebis & 180 & \multicolumn{2}{|c|}{ Lait de vache non décelé } & \\
\hline $4 \%$ vache pasteurisé & 180 & 0,093 & 0,018 & 5 \\
\hline $7 \%$ vache pasteurisé & 180 & 0,136 & 0,025 & 7 \\
\hline Pur brebis & 365 & \multicolumn{2}{|c|}{ Lait de vache non décelé } & \\
\hline $4 \%$ vache pasteurisé & 365 & 0,084 & 0,027 & 4 \\
\hline
\end{tabular}

(a) Moyenne de 9 valeurs. Mean of 9 values.

(b) Déterminé d'après l'abaque témoin. Determined from reference curve.

jours, ce taux est voisin de $50 \%$ pour la plupart des échantillons; il augmente ensuite plus doucement, et dépasse $60 \%$ pour les fromages fabriqués depuis 1 an.

\section{Isoélectrofocalisation. Détermination du pourcentage de lait de vache dans les fromages}

Les profils d'IEF des fromages Roquefort (temps d'affinage correspondant à 70, 140, 180 et 365 jours après la fabrication), et des fromages témoins sont représentés sur les figures 3 et 4 .

Parmi les 3 bandes de l'échantillon témoin $100 \%$ brebis, la bande $B$ choisie pour caractériser l'espèce brebis est la bande inférieure. La bande V caractérisant l'espèce vache est la plus basse des 2 bandes majeures de l'échantillon témoin $100 \%$ vache. Nous remarquons que quel que soit le temps d'affinage, les profils d'IEF sont similaires, et dans tous les cas, nous repérons sans difficulté les bandes $V$ et $\mathrm{B}$. 


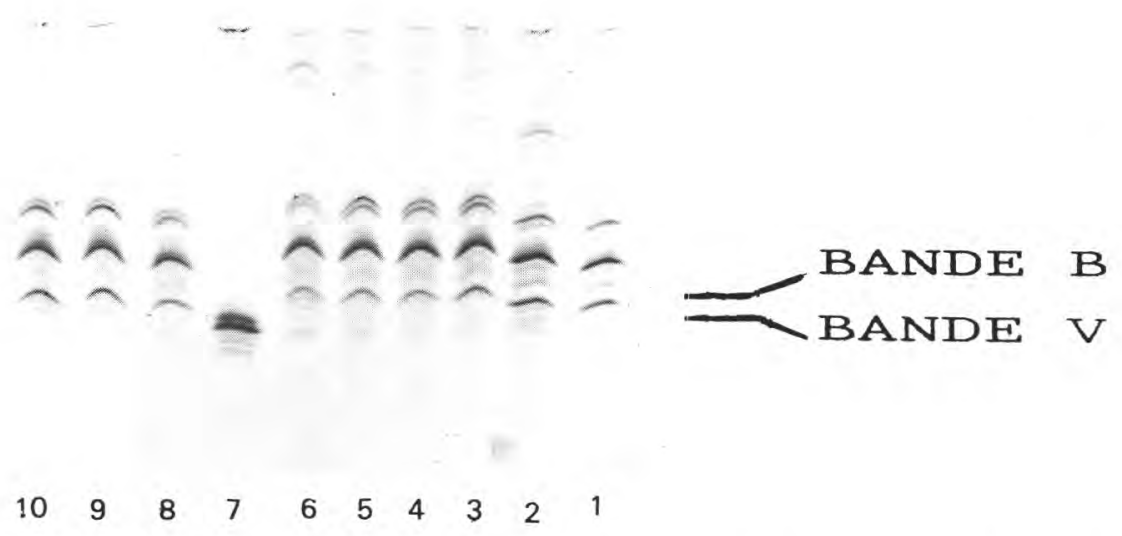

Fig 3. Profils d'IEF des lactosérums des fromages (de droite à gauche) : 1) témoin $100 \%$ brebis; 2) témoin $5 \%$ vache; 3) Roquefort $100 \%$ brebis, 70 jours; 4) Roquefort fraudé $4 \%$ vache pasteurisé 70 jours; 5) Roquefort fraudé $5 \%$ vache poudre, 70 jours; 6 ) Roquefort fraudé $7 \%$ vache pasteurisé, 70 jours; 7) témoin $100 \%$ vache; 8 ) Roquefort $100 \%$ brebis, 180 jours; 9) Roquefort fraudé $4 \%$ vache pasteurisé, 180 jours; 10 ) Roquefort fraudé $7 \%$ vache pasteurisé, 180 jours.

Isoelectric focusing patterns of wheys from cheeses (right to left) : 1), reference cheese, $100 \%$ ewe's milk; 2), reference cheese, $5 \%$ cow's milk; 3), Roquefort cheese, $100 \%$ ewe's milk, 70 d; 4) Roquefort cheese adulterated with $4 \%$ pasteurized cow's milk, $70 \mathrm{~d}$; 5), Roquefort cheese adulterated with $5 \%$ dried cow's milk, $70 \mathrm{~d}$; 6), Roquefort cheese adulterated with $7 \%$ pasteurized cow's milk, $70 \mathrm{~d}$; 7), reference cheese $100 \%$ cow's milk; 8), Roquefort cheese, 100\% ewe's milk, 180 d; 9), Roquefort cheese adulterated with $4 \%$ pasteurized cow's milk, $180 \mathrm{~d}$; 10), Roquefort cheese adulterated with $7 \%$ pasteurized cow's milk, $180 \mathrm{~d}$.

Les diagrammes des lectures densitométriques des fromages à 180 jours et du fromage témoin $10 \%$ vache sont représentés sur la figure 5 .

Les rapports de densité de bande v/b obtenus (moyenne des 9 valeurs - écart type), ainsi que les pourcentages de lait de vache correspondant déterminés sur l'abaque (fig 2) sont regroupés dans le tableau II. Pour les fromages contenant $4 \%$ de lait de vache (crus ou pasteurisés), et les fromages contenant $5 \%$ de lait de vache (poudre), nous évaluons la fraude à 3 ou $5 \%$ selon les cas. Pour les fromages contenant $7 \%$ de lait de vache, nous trouvons dans tous les cas des valeurs de $7 \%$. 


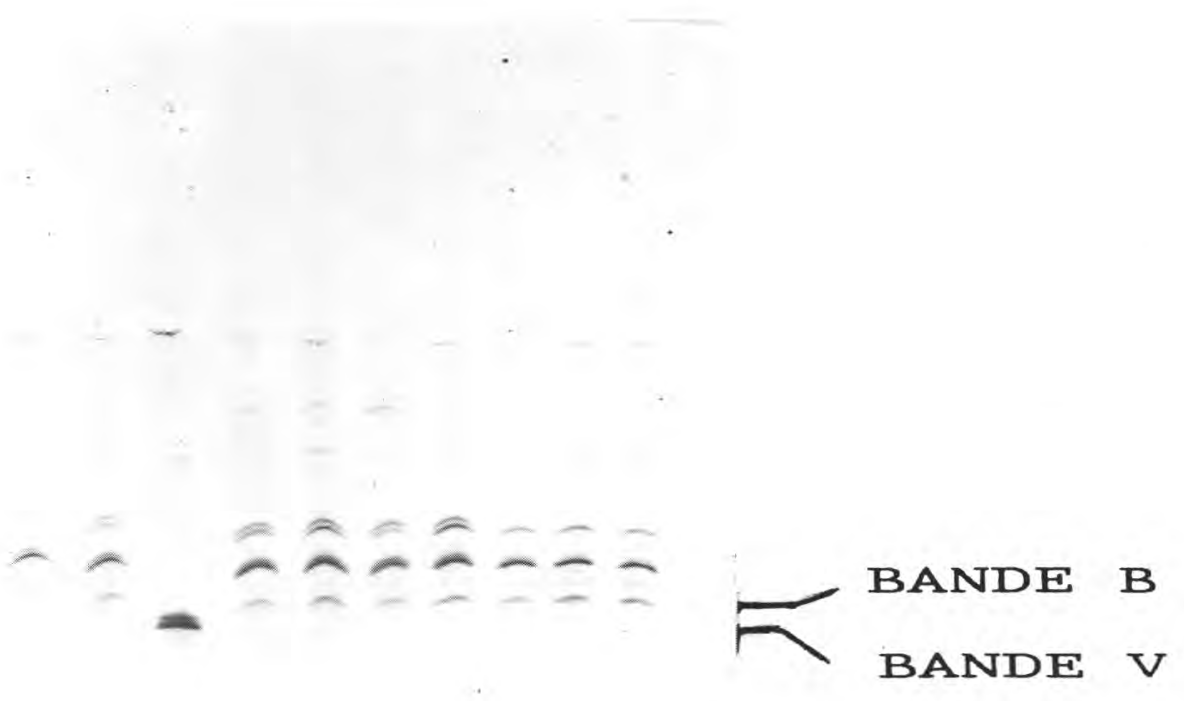

10

$\begin{array}{lllllllll}9 & 8 & 7 & 6 & 5 & 4 & 3 & 2 & 1\end{array}$

Fig 4. Profils d'IEF des lactosérums des fromages (de droite à gauche) : 1) témoin $100 \%$ brebis; 2) témoin $5 \%$ vache; 3 ) témoin $10 \%$ vache; 4) Roquefort $100 \%$ brebis, 140 jours; 5 ) Roquefort fraudé $4 \%$ vache cru, 140 jours; 6 ) Roquefort fraudé $5 \%$ vache poudre, 140 jours; 7 ) Roquefort fraudé $7 \%$ vache pasteurisé, 140 jours; 8 ) témoin $100 \%$ vache; 9) Roquefort $100 \%$ brebis, 365 jours; 10 ) Roquefort $4 \%$ vache pasteurisé, 365 jours.

Isoelectric focusing patterns of wheys from cheeses (right to left) : 1), reference cheese, $100 \%$ ewe's milk; 2) reference cheese, 5\% cow's milk; 3), reference cheese, 10\% cow's milk; 4), Roquefort cheese, $100 \%$ ewe's milk, 140 d; 5), Roquefort cheese adulterated with $4 \%$ raw cow's milk, 140 d; 6), Roquefort cheese adulterated with 5\% dried cow's milk, 140 d; 7), Roquefort cheese adulterated with 7\% pasteurized cow's milk, 140 d; 8), reference cheese, 100\% cow's milk; 9), Roquefort cheese, $100 \%$ ewe's milk, $365 d$; 10), Roquefort cheese adulterated with $4 \%$ pasteurized cow's milk, $365 d$.

\section{DISCUSSION}

L'analyse de la fraction azotée montre que les fromages Roquefort sont déjà fortement protéolysés à 70 jours. Malgré cet important degré de protéolyse, l'IEF des lactosérums nous a permis de déceler le lait de vache dans tous les fromages analysés, et ceci jusqu'à un an de maturation. En effet, quel que soit le temps d'affinage, la bande $\mathrm{V}$, absente sur le profil d'IEF des échantillons de Roquefort $100 \%$ brebis, est aisément repérable pour tous les fromages fraudés. La bande protéique choisie pour caractériser la présence de lait de vache n'est donc pas confondue avec une nouvelle bande, comme c'est le cas pour l'IEF des paracaséines $\kappa$ appliquée aux fromages type Roquefort âgés de plus d'un mois (Mauriello et al, 1989). 


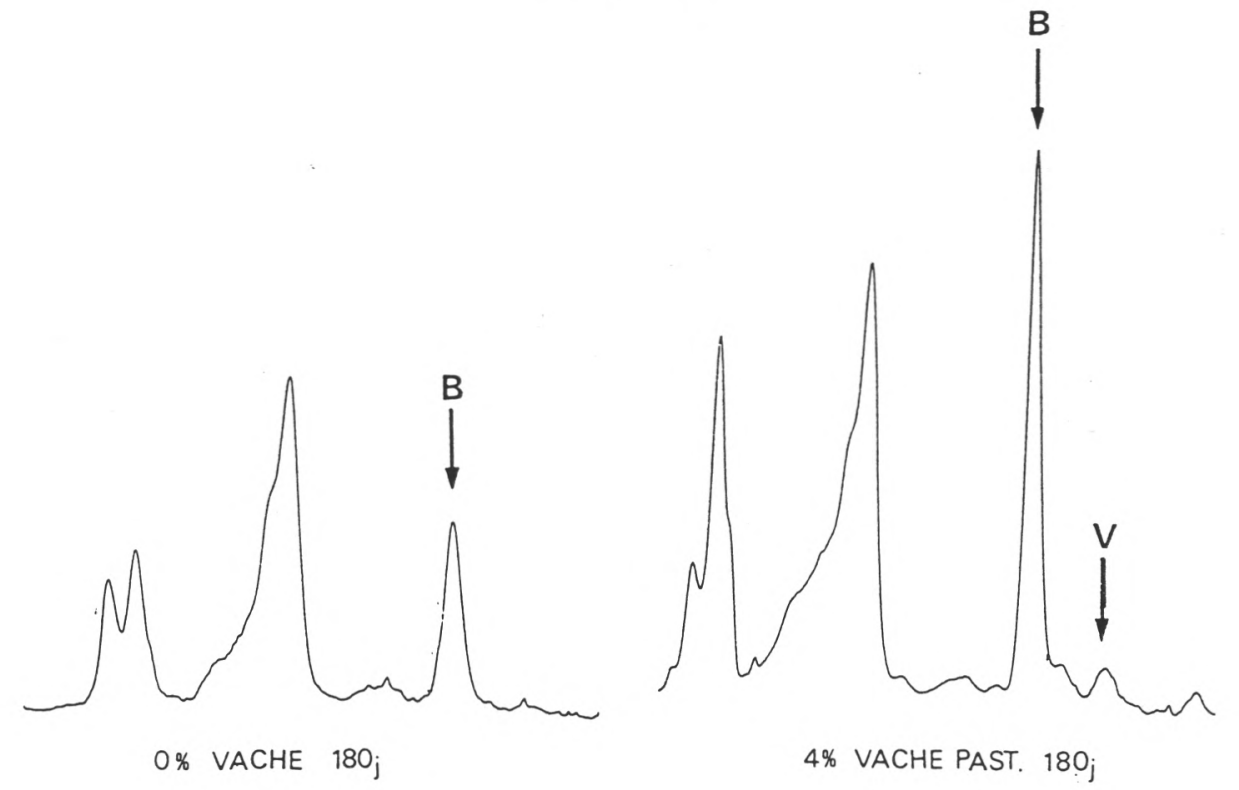

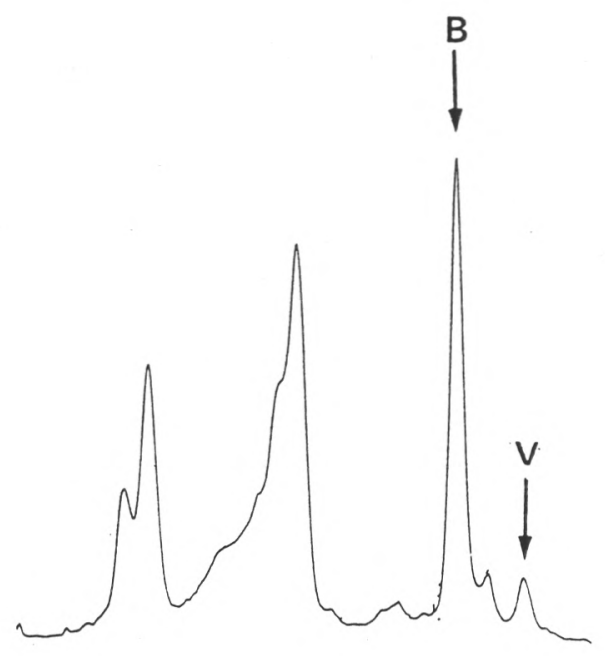

$7 \%$ VACHE PAST. $180_{i}$

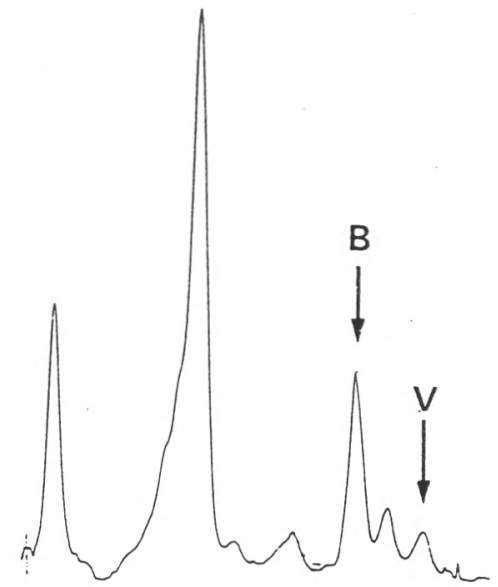

TEMOIN $10 \%$ VACHE

Fig 5. Lectures densitométriques (à $600 \mathrm{~nm}$ en transmission) des bandes IEF des fromages Roquefort à 180 jours.

Densitometric readings (600 nm transmission) of IEF patterns from Roquefort cheeses at $180 \mathrm{~d}$. 
En ce qui concerne l'analyse quantitative, la comparaison entre les teneurs réelles en lait de vache et les pourcentages déterminés sur la courbe montre que dans le cas présent, l'écart maximal obtenu est de 2 points (valeur trouvée de $3 \%$ pour une teneur réelle de $5 \%$ ). Cet écart est comparable à la marge d'erreur qu'on peut admettre pour le dosage du lait de vache dans les fromages de chèvre par électrophorèse des caséines (Pierre, 1977).

L'application de notre protocole basé sur l'IEF des lactosérums devra être encore élargie, notamment en complétant la gamme témoin par des valeurs inférieures à $5 \%$ et en analysant d'autres types de fromages (fromages fabriqués avec des laits ayant subi des traitements thermiques sévères).

Cependant, l'utilisation de l'abaque précédemment établi permet d'évaluer la quantité de lait de vache dans des fromages très protéolysés, type Roquefort.

\section{CONCLUSION}

Ce travail permet de conclure que la détermination qualitative et quantitative du lait de vache par isoélectrofocalisation des lactosérums est applicable aux cas des fromages très protéolysés.

En effet, l'ajout de lait de vache (cru, pasteurisé, poudre) a été mis en évidence dans des fromages par ailleurs difficiles à analyser par des techniques électrophorétiques basées sur les caséines (fromages type Roquefort jusqu'à 1 an).

Les méthodes s'intéressant aux protéines sériques peuvent donc présenter un intérêt pour l'étude des fromages à forte protéolyse; néanmoins, la validité du protocole en cas de traitement thermique sévère devra être étudiée.

\section{RÉFÉRENCES}

Addeo F, Anelli G, Stingo C, Chianese L, Petrilli P, Scudiero A (1984) Riconoscimento e dosaggio del latte bovino nel formaggio "Pecorino". Latte 9, 135-142

Blakesley RW, Boezi JA (1977) A new staining technique for proteins in polyacrylamide gels using Coomassie Brillant blue G 250. Anal Biochem 82, 580-582

Desmazeaud MJ, Gripon JC, Le Bars D, Bergère JL (1976) Étude du rôle des microorganismes et des enzymes au cours de la maturation des fromages. III. Influence des microorganismes (Streptococcus lactis, Penicillium caseicolum, Penicillium roqueforti). Lait 56, 379-396

Donovan M, Mulvihill DM (1987) Thermal denaturation and aggregation of whey proteins. Ir J Food Sci Technol 11, 87-100

Krause I, Belitz HD, Kaiser KP (1982) Detection of cow' milk in sheep's and goat's milk and cheese by isoelectric focusing in thin layers of polyacrylamide gels containing urea. $Z \mathrm{Le}$ bensm Unters Forsch 174, 195-199

Mauriello R, Chianese L, D'Acierno C, Kalatzopoulos G, Addeo F (1989) Riconoscimento e dosaggio del latte bovino nel formaggio Roquefort. Latte 14, 1071-1076

Pierre A (1977) Étude des facteurs influençant la précision de la méthode électrophorétique du dosage du lait de vache dans le lait et fromage de chèvre. Ann Falsif Expert Chim Toxicol 70, 213-222

Pierre A, Portmann A (1970) Emploi de l'électrophorèse en gel de polyacrylamide pour mettre en évidence et doser le lait de vache ajouté au lait de chèvre. Application au cas des fromages. Ann Technol Agric 19, 107130

Ramos M, Juarez M (1984) Update on existing analytical methods for detecting mixture of cow's, ewe's and goat's milk. Bull Fed Int Lait $181,1-9$

Rispoli S, Saugues R (1989) Isoélectrofocalisation des lactosérums de fromages de mélange brebis/vache sur gel de polyacrylamide. Application à la recherche et au dosage du lait de vache dans les fromages de brebis. Lait 69, 211-222 